\title{
Deglycosylated PD-L1 might be a better biomarker
}

PD-L1 expression is often used to determine the eligibility of patients for treatment with anti-PD-1 or anti-PD-L1 antibodies. Nonetheless, many trials reveal responses to these agents in patients with PD-L1-negative disease. Now, data from a retrospective study demonstrate that $N$-linked glycosylation of PD-L1, which interferes with antibody-based detection methods, might explain this inconsistency.

Lead author Mien-Chie Hung explains "According to previous studies, PD-L1 is a relatively small glycoprotein with heavy $N$-glycosylation on its extracellular domain". Therefore, "antibodies generated to recognize pure recombinant $\mathrm{PD}$-L1 peptide or protein antigens could have a differential binding affinity to the PD-L1 glycoprotein in human tissue owing to $N$-glycosylation".
To investigate this effect, researchers analysed PD-L1 expression before and after deglycosylation with peptide$N$-glycosidase F in pathological specimens from 200 patients with solid tumours, including breast, lung, colon, prostate and pancreatic cancers. A $>2$-fold increase in $\mathrm{H}$-score (reflecting PD-L1-positivity) was observed in $47.5 \%$ of samples following deglycosylation. Subsequent analysis of non-small-cell lung cancer specimens obtained from three independent cohorts with known PD-L1 tumour proportion scores (TPS) confirmed the clinical relevance of PD-L1 glycosylation: $22.5 \%$ and $16 \%$ of patients had increases in TPS from $<1 \%$ to $\geq 5 \%$ and $\geq 49 \%$, respectively, following deglycosylation - scores that would have made these patients eligible for treatment with nivolumab or pembrolizumab.

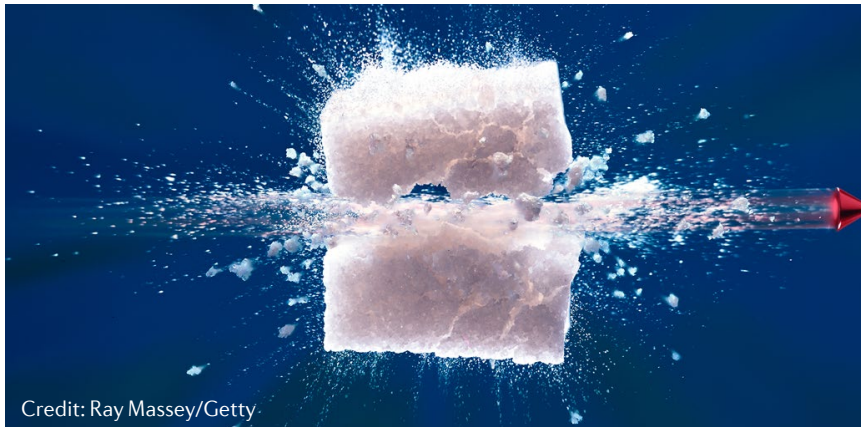

An analysis of samples from 95 patients who received anti-PD-1 or anti-PD-L1 antibodies revealed statistically significant positive correlations of both PD-L1 H score and PD-L1 TPS with overall survival after $(P=0.033$ and $P=0.005$, respectively) but not before $(P=0.80$ and $P=0.29)$ deglycosylation.

Hung summarizes "our method identified a population $(\sim 16 \%)$ of anti-PD-L1 responders whose PD-L1 status changed from negative to positive", adding that "deglycosylation might be an effective method of improving the predictive power of PD-L1 as a biomarker".

Peter Sidaway the predictive power of PD-L1 ...

\section{Ibrutinib outperforms FCR in CLL}

Ibrutinib has shown promising activity in patients with relapsed and/or refractory chronic lymphocytic leukaemia (CLL) as well as in those deemed unfit to receive other agents. "No study has previously compared first-line ibrutinib with the gold standard therapy (fludarabine, cyclophosphamide and rituximab (FCR)) in patients $\leq 70$ years of age," explains Tait Shanafelt, lead investigator of a study that has now shown improved outcomes with ibrutinib in this patient population.

In the E1912 trial, 354 patients were randomly assigned to receive ibrutinib plus rituximab and 175 patients to FCR. At 33.6 months of follow up, both progression-free survival (PFS) and overall survival (OS) were better with ibrutinib plus rituximab: 3-year PFS $89.4 \%$ versus $72.9 \%$ (HR 0.35 (95\% Cl 0.22-0.56); $P<0.001)$ and 3-year OS 98.8\% versus $91.5 \%$ (HR 0.17 (95\% Cl 0.05-0.54); $P<0.001$ ).

"Surprisingly, this advantage was apparent at the first interim analysis, resulting in the data safety monitoring board recommending immediate release of the results because they were deemed practice-changing," highlights Shanafelt.

Subgroup analyses of 3-year PFS results revealed that ibrutinib plus rituximab is superior to FCR in patients with non-mutated IGHV this advantage was apparent at the first interim analysis ... recommending immediate release of the results

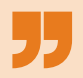
(90.7\% versus $62.5 \%$; HR 0.26 (95\% $\mathrm{Cl}$ 0.14-0.50)) but not in those with mutated IGHV (87.7\% versus $88.0 \%$; HR 0.44 (95\% Cl 0.14-1.36)), although this could change with further follow-up.

The overall response rate was higher with ibrutinib plus rituximab: $95.8 \%$ versus $81.1 \%$. However, the complete response and minimal residual disease negativity rates were lower in the ibrutinib plus rituximab group: $17.2 \%$ versus $30.3 \%$, and $8.3 \%$ versus $59.2 \%$, respectively, suggesting that, whereas ibrutinib extends survival, FCR results in deeper responses.

The incidence of grade $\geq 3$ adverse events (AEs) was similar in both groups $-80.1 \%$ with ibrutinib plus rituximab versus $79.7 \%$ with FCR - but the toxicity profiles were different for each regimen: the ibrutinib regimen was associated with lower incidence of neutropenia and infectious complications and a higher incidence of hypertension and haemorrhagic AEs.

These results indicate that ibrutinib should be considered a new standardof-care first-line treatment for patients with CLL $\leq 70$ years of age, although whether it needs to be combined with rituximab remains unclear. "Future trials are evaluating whether combining ibrutinib with other novel targeted treatments can lead to deeper remissions, eliminating the need for patients to take therapy indefinitely", mentions Shanafelt.

Diana Romero

ORIGINAL ARTICLE Shanafelt, T. D. et al. Ibrutinib-rituximab or chemoimmunotherapy for chronic lymphocytic leukemia. N. Engl.J. Med. 381, 432-443 (2019) 